\title{
Numerical Study of Unsteady MHD Pulsating Flow of Couple Stress Fluid through Porous Medium between Permeable Beds Using Generalized Differential Quadrature Method (GDQM)
}

\author{
I. M. Eldesoky ${ }^{1}$, M. H. Kamel $^{2}$ and R. M. Abumandour ${ }^{1}$ \\ ${ }^{1}$ Basic Engineering Sciences Department, Faculty of Engineering, Menofia University, Shebin El-Kom, Egypt \\ ${ }^{2}$ Engineering Mathematics and Physics Department, Faculty of Engineering, Cairo University, Egypt. \\ (E-mail: eldesokyi@yahoo.com)
}

Abstract - A numerical study of unsteady MHD pulsating flow of an incompressible Newtonian electrically conducting couple stress fluid through porous medium between permeable beds under the influence of periodic body acceleration. The couple stress fluid is injected into the channel from the lower permeable bed with a certain velocity and is sucked into the upper permeable bed with the same velocity. The flow between the permeable beds is assumed to be governed by couple stress fluid flow equations of Navier-Stokes and that in the permeable regions by Darcy's law. The slip condition plays an important role in shear skin, spurt, and hysteresis effects. The fluids that exhibit boundary slip have important technological applications such as in polishing valves of artificial heart and internal cavities. A numerical solution of the equation of motion is obtained by a new algorithm "modified generalized differential quadrature method (MGDQM)" which applying a generalized differential quadrature method (GDQM), to derivatives with respect to space variables of differential equations and for the time derivative applying $4^{\text {th }}$ order Runge Kutta Method. This combination of DQM and 
$4^{\text {th }}$ order RK method gives very good numerical technique for solving time dependent problems. The numerical results and the effects of the material parameters are presented and discussed through graphs.

Keywords - Pulsatile flow, MHD, couple stress fluid, permeable beds, body acceleration, porous medium, Differential quadrature method, Runge-Kutta method.

\section{INTRODUCTION}

The MHD fluid flow in channel is an interesting area in the study of fluid mechanics because of its relevance to various engineering applications. The MHD effects are widely exploited in different industrial processes ranging from metallurgy to the production pure crystals. A field in which MHD will play an essential role is nuclear fusion, where it is involved in at least two different problems: the confinement and dynamics of plasma, and the behaviour of the liquid metal alloys employed in some of the currently considered designs of tritium breeding blankets.

A fluid flow driven by a pulsatile pressure gradient through porous media in a channel or a pipe are very much pervalent in nature and hence their study is of principal interest in many scientific and engineering applications. This type of flows are of great importance in chemical engineering (for filteration and water purification processes) and petroleum engineering (for stuyding the movement of natural gas, oil and water through the oil resevoirs). Also, this type of flows are of great importance in physiology, biomedical engineering and it has biological applications in relation to hemodynamics, industrial applications in relation to heat exchange efficiency, applications in natural systems like circulatory systems, respiratory systems, vascular diseases, in engineering 
systems like reciprocating pumps, IC engines, combustors and applications in MEMS micro fluidic engineering applications [1-3].

Also, the fluid flow driven by a pulsatile pressure gradient through porous media in a channel or a pip has application in the dialysis of blood through artificial kidneys or blood flow in the lung alveolar sheet. Afifi and Gad [4] studied the flow of a Newtonian, incompressible fluid under the effect of transverse magnetic field through a porous medium between infinite parallel walls on which a sinusoidal traveling wave is imposed. Ramamurthy and Shanker [5] studied magneto-hydrodynamic effects on blood flow through a porous channel, they considered the blood as a Newtonian fluid and conducting fluid. Arterial MHD pulsatile flow of blood under periodic body acceleration has been studied by Das and Saha [6]. The effect of uniform transverse magnetic field on its pulsatile motion through an axi-symmetric tube is analyzed by Dulal and Ananda [7]. Chaturani and Palanisamy [8] discussed the flow characteristics of blood under external body acceleration. Eldesoky [9] studied the unsteady pulsatile flow of blood through porous medium in an artery under the influence of periodic body acceleration and slip condition in the presence of magnetic field considering blood as an incompressible electrically conducting fluid. An analytical solution of the equation of motion is obtained by applying the Laplace transform. Eldesoky [10] studied the influence of slip on the peristaltic flow in an axisymetric cylindrical tube. A compressible Maxwell fluid saturates the homogenous porous medium. Modified Darcy's law has been used to model the governing equation. The analytical solutions of the equation of motion are obtained by applying a perturbation analysis. Recently, Eldesoky, Kamel, Hussien and Abumandour [11] studied the unsteady pulsatile flow through porous medium in an artery under the influence of periodic body acceleration in the presence of magnetic field numerically using Generalized Differential 
Quadrature Method (GDQM). Eldesoky [12] studied the influence of relaxation time on MHD pulsatile flow of blood through porous medium in an artery under the effect of periodic body acceleration in the presence of magnetic field considering blood as an incompressible electrically conducting fluid. An analytical solution of the equation of motion is obtained by applying the Laplace Transform. Wang [13] studied the pulsatile flow of a viscous fluid in a porous channel. Vajravelu et al. [14] studied the pulsatile flow of a viscous fluid between permeable beds.

The boundary conditions for the flow through porous beds need special attention. Generally the no-slip condition is valid on the boundary when a fluid flows between impermeable surfaces. But when it flows between permeable surfaces, the no-slip condition is no longer valid since there will be a migration of fluid, tangential to the boundary within the permeable surfaces. The velocity within the permeable beds will be different from the velocity of the fluid in the channel and we have to match the two velocities at the interface. A simple theory presented by Beavers and Joseph in [15] based on replacing the effect of the boundary layer with a slip velocity proportional to the exterior velocity gradient is proposed and shown to be in reasonable agreement with experimental results.

The theory of couple stress fluids initiated by Stokes [16] is a generalization of the classical theory of viscous fluids, which allows for the presence of couple stresses and body couples in the fluid medium, which studied a series of boundary-value problems are solved to indicate the effects of couple stresses. A striking feature of this model is that it results in equations that are similar to the Navier Stokes equations, there by facilitating a comparison with the results for the classical non polar case. An excellent introduction to this theory written by Stokes [17] in which he has presented a detailed account of couple stress fluids. This 
theory has several industrial and scientific applications as well, which comprise pumping fluids such as synthetic fluids, polymer thickened oils, liquid crystal, animal blood, synovial fluid present in synovial joints and the theory of lubrication [18-23]. Some salient references to couple stress flows through tubes and channels can be seen in Stokes [17]. Several flows past axisymmetric bodies dealing with couple stress fluids have been studied by Rao and Iyengar [24] and Srinivasacharya [25]. Rao and Iyengar [24] have made analytical and computational studies of diverse couple stress fluid flows dealing with a class of axisymmetric problems. EL-Dabe et.al [26] discussed the effects of couple stresses on pulsatile hydro magnetic Poiseuille flow, using perturbation technique. Srivastava [27] studied the effects of an axially symmetric mild stenosis on the flow of blood, when blood is represented by a couple stress fluid. He also studied the peristaltic transport of a couple stress fluid under a zero Reynolds number and long wavelength approximation [28]. Naduvinamani et al. studied a number of problems with couple stress fluid between porous journal bearings and porous rectangular plates [2931]. Devakar and Iyengar [32, 33] studied Stokes Problems for an incompressible couple stress fluid under isothermal conditions and run up flow of a couple stress fluid between parallel plates using Laplace transform technique. Radhika and Iyengar [34] studied the Stokes flow of an incompressible couple stress fluid past a porous spheriodal shell.

Numerical approximation methods for solving partial differential equations have been widely used in various engineering fields. Classical techniques such as finite elements and finite differences methods are well developed and well known. These methods can provide very accurate results by using a large number of grid points. In seeking an alternate numerical method using fewer grid points to find results with acceptable accuracy, the method of DQM was introduced by [36-45]. 
In this paper, modified GDQM used for studying the unsteady pulsating flow of an incompressible couple stress fluid between permeable beds through Porous Medium in channel under the influence of periodic body acceleration in the presence of magnetic field. Modified GDQM is a new algorithm which applying a generalized differential quadrature method (GDQM), to derivatives with respect to space variables of differential equations and for the time derivative applying $4^{\text {th }}$ orders Runge Kutta Method. This combination of DQM and $4^{\text {th }}$ order RK method gives very good numerical technique for solving time dependent problems. Stability of $4^{\text {th }}$ order RKM criterias are controlled with several values of time increment $\Delta t$ and number of grid points $N$ in space region. The fluid is driven by an unsteady pressure gradient through the permeable beds. The flow through the permeable beds is assumed to be governed by Darcy's law and the flow between permeable beds by couple stress fluid flow equations of Stokes. The equations are solved numerically and the numerical results are presented and discussed through graphs. We observe that our results are in good agreement with those obtained by Vajravelu et al. [14] in the respective special cases considered by them.

\section{MATHEMATICAL FORMULATION}

We consider the pulsatile flow of an incompressible couple stress fluid between two permeable beds. The fluid is injected into the channel from the lower permeable bed with a velocity $V$ and is sucked into the upper permeable bed with the same velocity. The flow between the permeable beds is assumed to be governed by couple stress fluid flow equations Stokes [17]. 
Let the $x$-axis be taken along the interface and the $y$-axis perpendicular to it. Let $y=0$ and $y=h$ represent the interfaces of the permeable beds under consideration (see figure 1).

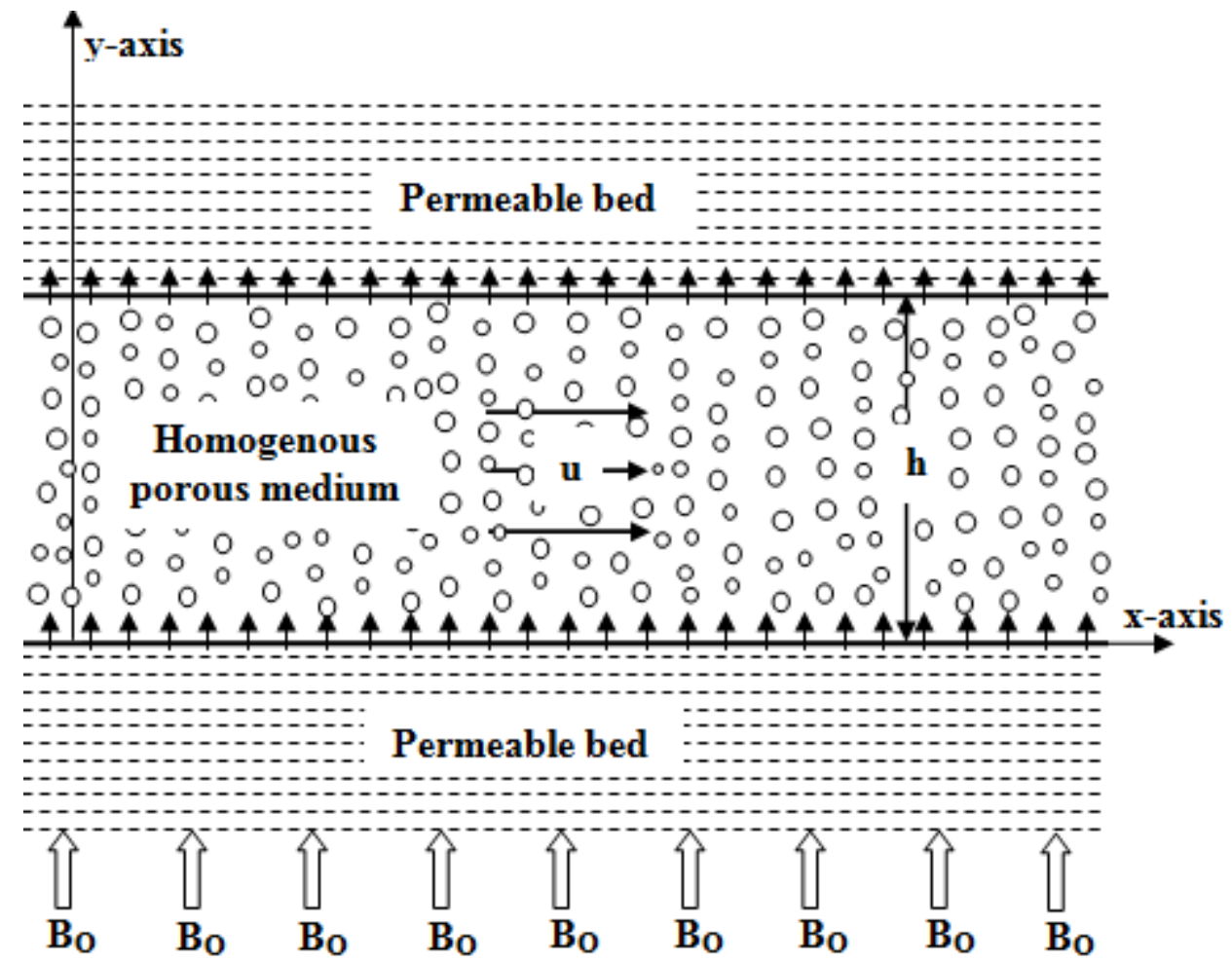

Figure 1: Flow diagram for the flow

The following assumptions are made in the analysis of the problem; the permeable beds are homogeneous, the flow as axially symmetric, laminar, pulsatile and fully developed, and the pressure gradient and body acceleration $G$ are given by:

$$
\begin{array}{rlrl}
-\frac{1}{\rho} \frac{\partial p}{\partial x}=A_{O}+A_{1} \cos \left(\omega_{p} t\right), & & t \geq 0, \\
G=a_{O} \cos \left(\omega_{b} t\right), & t \geq 0
\end{array}
$$

where $A_{o}$ and $A_{I}$ are pressure gradient of steady flow and amplitude of oscillatory part respectively, $a_{\boldsymbol{O}}$ is the amplitude of the body acceleration, $\omega_{p}=2 \pi f_{p}, \omega_{b}=2 \pi f_{b}$ with $f_{p}$ is the pulse frequency, and $f_{b}$ is the body acceleration frequency and $t$ is time.

Under the above mentioned assumption, the field equations describing a couple stress fluid flow [17] are: 


$$
\begin{gathered}
\frac{\partial \rho}{\partial t}+\operatorname{div}(\rho \bar{q})=0 \\
\rho \frac{\partial \bar{q}}{\partial t}=\rho \bar{f}+\frac{1}{2} \operatorname{curl}(\rho \bar{c})-\operatorname{grad}(p)-\mu \operatorname{curl}(\operatorname{curl}(\bar{q})) \\
-\eta \operatorname{curl}(\operatorname{curl}(\operatorname{curl}(\operatorname{curl}(\bar{q}))))+(\lambda+2 \mu) \operatorname{grad}(\operatorname{div}(\bar{q}))
\end{gathered}
$$

where $\rho$ is the density of the fluid, $\bar{q}$ is the velocity vector, $p$ is the fluid pressure and $\bar{f}, \bar{c}$ are the body force per unit mass and body couple per unit mass respectively. The quantities $\lambda$ and $\mu$ are the viscosity coefficients and $\eta, \eta^{\prime}$ are the couple stress viscosity coefficients satisfying the constraints

$$
\mu \geq 0, \quad 3 \lambda+2 \mu \geq 0, \quad \eta \geq 0,\left|\eta^{\prime}\right| \leq \eta
$$

There is a length parameter $l=\sqrt{\frac{\eta}{\mu}}$ which is a characteristic measure of the polarity of the couple stress fluid and this parameter is identically zero in the case of non polar fluids.

Under the assumption made, we have $\bar{q}=(u(y, t), V, 0)$.

$$
\begin{gathered}
\frac{\partial u}{\partial x}+\frac{\partial v}{\partial y}=0 \\
\frac{\partial u}{\partial t}+V \frac{\partial u}{\partial y}=-\frac{1}{\rho} \frac{\partial p}{\partial x}+\bar{G}+\frac{\mu}{\rho} \frac{\partial^{2} u}{\partial y^{2}}-\frac{\eta}{\rho} \frac{\partial^{4} u}{\partial y^{4}}-\left(\frac{\mu}{\rho k}\right) u+\frac{\bar{J} \times \bar{B}}{\rho} \\
0=\frac{\partial p}{\partial y}
\end{gathered}
$$

Maxwell's generalized electromagnetic field equations are

$$
\bar{\nabla} \cdot \bar{B}=0, \quad \bar{\nabla} \cdot \bar{D}=\rho, \quad \bar{\nabla} \times \bar{B}=\mu_{O} \bar{J}, \quad \bar{\nabla} \times \bar{E}=-\frac{\partial \bar{B}}{\partial t} .
$$

Ohm's law is

$$
\bar{J}=\sigma(\bar{E}+\bar{q} \times \bar{B}) .
$$

where $\mu_{O}$ the fluid magnetic permeability, $\bar{B}=\left(0, B_{O}, 0\right)$ the magnetic field vector, $\bar{E}$ the electric field vector, $\bar{J}$ the current density vector, $k$ is the permeability parameter of porous medium, $\sigma$ the fluid electric 
conductivity and $D$ the electric flux density. For small magnetic Reynolds number, the linearlized magnetohydrodynamic force $\bar{J} \times \bar{B}$ can be put into the following form:

$\mathrm{D}$ and $\mathrm{B}$ are the electric and magnetic flux densities

$$
\overline{\boldsymbol{J}} \times \overline{\boldsymbol{B}}=-\sigma \boldsymbol{B}_{O}^{2} \boldsymbol{u} .
$$

Under the above assumptions the equation of motion (7) is

$$
\begin{aligned}
\frac{\partial u}{\partial t}+V \frac{\partial u}{\partial y}= & A_{O}+A_{1} \cos \left(\omega_{p} t\right)+a_{O} \cos \left(\omega_{b} t\right)+\frac{\mu}{\rho} \frac{\partial^{2} u}{\partial y^{2}}- \\
& \frac{\eta}{\rho} \frac{\partial^{4} u}{\partial y^{4}}-\left(\frac{\mu}{\rho k}\right) u+\frac{\sigma B_{O}^{2} u}{\rho}
\end{aligned}
$$

For the slip condition $u_{t}=A_{p} \partial u_{t} / \partial n$, where $u_{t}$ is the tangential velocity, $n$ is normal to the surface, and $A_{p}$ is a coefficient close to the mean free path of the molecules of the fluid [35]. Than the boundary conditions that must be satisfied by the fluid on the wall of channel are:

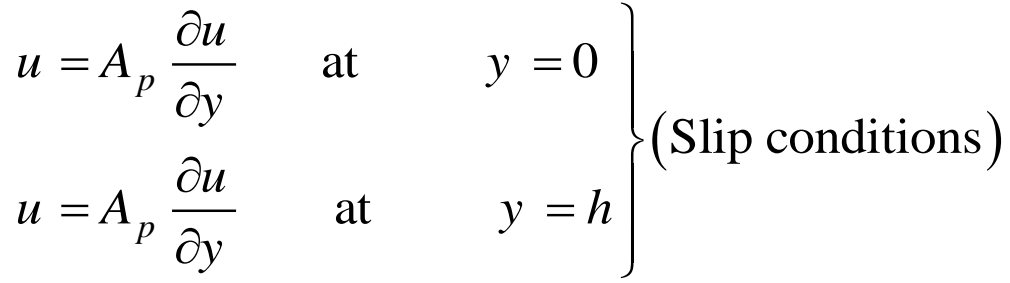

$$
\begin{aligned}
& \left.\left.\begin{array}{lll}
\frac{\partial^{2} u}{\partial y^{2}}=0 \quad \text { at } & y=0 \\
\frac{\partial^{2} u}{\partial y^{2}}=0 & \text { at } & y=h
\end{array}\right\} \text { (Vanishing of couple stresses }\right)
\end{aligned}
$$

Let us introduce the following dimensionless quantities:

$$
\begin{aligned}
& u^{*}=\frac{u}{\omega h}, \quad t^{*}=\frac{t v}{h^{2}}, \quad A_{O}^{*}=\frac{\rho h}{\mu \omega} A_{O}, \quad A_{1}^{*}=\frac{\rho h}{\mu \omega} A_{1}, \\
& a_{O}^{*}=\frac{\rho h}{\mu \omega} a_{O}, \quad k^{*}=\frac{k}{h^{2}}, \quad b=\frac{\omega_{b}}{\omega_{p}}, \quad y^{*}=\frac{y}{h} .
\end{aligned}
$$

In terms of these variables, equation (12) after dropping the stars becomes: 


$$
\begin{aligned}
\frac{\partial u}{\partial t}= & A_{O}+A_{1} \cos (t)+a_{O} \cos (b t)-\frac{1}{\beta^{2}} \frac{d^{4} u}{d y^{4}}+\frac{d^{2} u}{d y^{2}}- \\
& R \frac{d u}{d y}-\left(H_{a}{ }^{2}+\frac{1}{k}\right) u .
\end{aligned}
$$

Also, the boundary conditions equation (13) becomes:

$$
\left.\begin{array}{llrl}
u=k_{n} \frac{d u}{d y} & \text { at } & y=0 \\
u=k_{n} \frac{\partial u}{\partial y} & \text { at } & y=1
\end{array}\right\} \text { (Slip conditions) }
$$

And the initial condition is

$$
u(y, 0)=1 \quad \text { at } \quad t=0
$$

Where the Hartmann number, $H a$, the Reynolds number, $R$, the Couple stress parameter $\beta$ and the Knudsen number $k_{n}$ are defined respectively by

$$
H_{a}=B_{O} h \sqrt{\frac{\sigma}{\mu}}, \quad R=\frac{\rho V h}{\mu}, \quad \beta^{2}=\frac{h^{2} \mu}{\eta}, \quad k_{n}=\frac{A_{p}}{h} .
$$

\section{Generalized Differential Quadrature Method (GDQM)}

The DQM is a numerical solution technique for initial and/or boundary value problems. This technique has been successfully employed in a variety of problems in engineering and physical sciences. The DQM approximates the derivative of a function at any location by a linear summation of all the functional values along a mesh (grid) line. In order to overcome the deficiencies which appears in classical DQM, Bellman et al. [36, 37], a generalized differential quadrature (GDQ), which was recently proposed by Shu and Richards [39-42] for solving partial differential equations in fluid mechanics, vibration analysis and structural 
analysis. The technique of GDQM for the solution of partial differential equations extended and generalized. Numerical examples have shown the super accuracy, efficiency, convenience and the great potential of this method. The GDQM, which was recently proposed by [39-41] for solving partial differential equations. For the discretization of the first and higher order derivatives, the following linear constrained relationships are applied

$$
f_{x}^{(n)}\left(x_{i}, t\right)=\sum_{j=1}^{N} C_{i j}^{(n)} f\left(x_{j}, t\right), \quad n=1,2, \ldots, N-1,
$$

for $i=1,2, \ldots, N$; where $f_{x}^{(n)}$ indicate $n^{\text {th }}$ order derivatives of $f(x, t)$ with respect to $x$ at $x_{i}, N$ is the number of grid points in the whole dominant $C_{i j}^{(n)}$ are the weighting coefficients. The key to DQ is to determine the weighting coefficients for the discretization of a derivative of any order. In order to find a simple algebraic expression for calculating the weighting coefficients without restricting the choice of grid meshes, [3941] gave a convenient and recurrent formula for determining the derivative weighting coefficients.

To determine the weighting coefficients of the GDQ method as:

Weighting coefficients for the first order derivative

$$
\begin{array}{rlrl}
C_{i j}^{(1)} & =\frac{M_{N}^{(1)}\left(x_{i}\right)}{\left(x_{i}-x_{j}\right) \cdot M_{N}^{(1)}\left(x_{j}\right)}, & i, j=1,2, \cdots, N \quad \text { and } \quad i \neq j \\
C_{i i}^{(1)}=-\sum_{j=1, j \neq i}^{N} C_{i j}^{(1)}, & i=1,2, \cdots, N
\end{array}
$$

Where

$$
\begin{aligned}
& M(x)=\left(x-x_{1}\right)\left(x-x_{2}\right) \cdots\left(x-x_{N}\right) \\
& M^{(1)}\left(x_{i}\right)=\prod_{k=1, k \neq i}^{N}\left(x_{i}-x_{k}\right)
\end{aligned}
$$

Weighting coefficients for the second and higher order derivatives 


$$
\begin{aligned}
C_{i j}^{(n)}= & n\left(C_{i j}^{(1)} C_{i i}^{(n-1)}-\frac{C_{i j}^{(n-1)}}{x_{i}-x_{j}}\right), \\
& \quad \text { for } j \neq i, \quad i, j=1,2, \ldots, N ; \quad n=2,3, \ldots, N-1 . \\
C_{i i}^{(n)}= & -\sum_{j=1 . j \neq i}^{N} C_{i j}^{(n)}, \quad \text { for } \quad i, j=1,2, \ldots, N ; \quad n=2,3, \ldots, N-1 .
\end{aligned}
$$

Where $C_{i j}^{(n)}$ and $C_{i j}^{(n-1)}$ are the weighting coefficients of the $n^{\text {th }}$ and the $(n-1)^{t h}$ derivatives. Thus equations (23) and (24) together with equations (20) and (21) give a convenient and general form for determining the weighting coefficients for the derivatives of orders one through $N-1$.

\section{Numerical discretization and stability of the scheme}

In the present study, substituting the DQ derivative approximations given in equation (19) in the governing equation (15) and boundary conditions equation (16). The coordinates of the grid points are chosen according to Chebyshev-Gauss-Lobatto by using $N$ sampling as:

$$
X(i)=\frac{1}{2}\left[1-\cos \left(\frac{i-1}{N-1} \pi\right)\right], \quad i=1,2,3, \ldots, N ;
$$

The GDQM is applied for the discretization of space derivatives of the unknown function $u$.

$$
\begin{gathered}
\frac{\partial u\left(y_{i}, t\right)}{\partial t}=A_{O}+A_{1} \cos (t)+a_{O} \cos (b t)-\frac{1}{\beta^{2}} \sum_{j=1}^{N} C_{i, j}^{(4)} \cdot u_{j}+\sum_{j=1}^{N} C_{i, j}^{(2)} \cdot u_{j}- \\
R \sum_{j=1}^{N} C_{i, j}^{(1)} \cdot u_{j}-\left(H_{a}{ }^{2}+\frac{1}{k}\right) u_{i} . \quad i=1,2, \cdots, N
\end{gathered}
$$

Where $u_{i}, i=1,2, \ldots, N$; is the velocity value at the grid $y_{i}, C_{i j}^{(1)}$, $C_{i j}^{(2)}$ and $C_{i j}^{(4)}$ are the weighting coefficient matrixes of the first, second and forth order derivatives. Similarly, the derivatives in the boundary conditions can be discretized by the GDQM. As a result, the numerical boundary conditions can be written as: 


$$
\left.\begin{array}{l}
u_{1}=k_{n} \sum_{k=1}^{N} C_{1, k}^{(1)} \cdot u_{k} \\
u_{N}=k_{n} \sum_{k=1}^{N} C_{N, k}^{(1)} \cdot u_{k}
\end{array}\right\}
$$

(Vanishing of couple stresses)

Equation (26) can't be easily substituted into the governing equation. However, we can give solutions, $u_{1}, u_{2}, u_{N-1}$ and $u_{N}$ as:

$$
\begin{aligned}
& u_{1}=R_{36} \cdot \sum_{k=3}^{N-2} C_{1, k}^{(1)} \cdot u_{k}+R_{37} \cdot \sum_{k=3}^{N-2} C_{N, k}^{(N)} \cdot u_{k}+R_{38} \cdot \sum_{k=3}^{N-2} C_{1, k}^{(2)} \cdot u_{k}+R_{39} \cdot \sum_{k=3}^{N-2} C_{N, k}^{(2)} \cdot u_{k} \\
& u_{2}=R_{24} \cdot \sum_{k=3}^{N-2} C_{1, k}^{(1)} \cdot u_{k}+R_{26} \cdot \sum_{k=3}^{N-2} C_{N, k}^{(N)} \cdot u_{k}+R_{25} \cdot \sum_{k=3}^{N-2} C_{1, k}^{(2)} \cdot u_{k}+R_{27} \cdot \sum_{k=3}^{N-2} C_{N, k}^{(2)} \cdot u_{k} \\
& u_{N-1}=R_{20} \cdot \sum_{k=3}^{N-2} C_{1, k}^{(1)} \cdot u_{k}+R_{21} \cdot \sum_{k=3}^{N-2} C_{N, k}^{(N)} \cdot u_{k}+R_{22} \cdot \sum_{k=3}^{N-2} C_{1, k}^{(2)} \cdot u_{k}+R_{23} \cdot \sum_{k=3}^{N-2} C_{N, k}^{(2)} \cdot u_{k} \\
& u_{N}=R_{48} \cdot \sum_{k=3}^{N-2} C_{1, k}^{(1)} \cdot u_{k}+R_{49} \cdot \sum_{k=3}^{N-2} C_{N, k}^{(N)} \cdot u_{k}+R_{50} \cdot \sum_{k=3}^{N-2} C_{1, k}^{(2)} \cdot u_{k}+R_{51} \cdot \sum_{k=3}^{N-2} C_{N, k}^{(2)} \cdot u_{k}
\end{aligned}
$$

According to equation (27), $u_{1}, u_{2}, u_{N-1}$ and $u_{N}$ is expressed in terms of $u_{3}, u_{4}, \cdots, u_{N-2}$, and can be easily substituted into the governing equation (25). It should be noted that equation (26) provides four boundary equations. In total we have $N$ unknowns $u_{1}, u_{2}, \cdots, u_{N}$. In order to close the system, the discretized governing equation (25) has to be applied at $N-4$ mesh points. This can be done by applying equation (25) at grid points $y_{3}, y_{4}, \ldots, y_{N-2}$. Substituting equations (27) into equation (25) gives:

$$
\frac{\partial u\left(y_{i}, t\right)}{\partial t}=A_{O}+A_{1} \cos (t)+a_{O} \cos (b t)-\frac{1}{\beta^{2}} \sum_{j=3}^{N-2} C_{i, j}^{(4)} \cdot u_{j}+\sum_{j=3}^{N-2} C_{i, j}^{(2)} \cdot u_{j}-
$$




$$
R \sum_{j=3}^{N-2} C_{i, j}^{(1)} \cdot u_{j}-\left(H_{a}{ }^{2}+\frac{1}{k}\right) u_{i} \cdot \quad i=3,4, \cdots, N-2
$$

It is noted that equation (28) has $N-4$ equations with $N-4$ unknowns.

Now, the discretization for time derivative will be performed by using Runge-Kutta $4^{\text {th }}$ order Method. Now, $\frac{\partial u}{\partial t}$ is also considered discretized as $\frac{\partial u_{i j}}{\partial t}$, thus equation (28) is a set of DQ algebraic equations which can be written in a matrix form

$$
[A]\{u\}=\{b\},
$$

Where $\{u\}=\left\{u_{3}, u_{4}, \cdots, u_{N-2}\right\}$ is a vector of unknown $N-4$ functional values at all discretized points of the region, $[A]$ is the $(N-4) \times(N-4)$ coefficient matrix, and the right hand side vector $\{b\}$ of size $(N-4) \times 1$ contains first order time derivatives of the function $u$ at the same discretized points. Therefore a numerical scheme is necessary for handling these time derivatives. Equation (29) can be solved by several time integration schemes such as Euler, Modified Euler, and Runge-Kutta $4^{\text {th }}$ order Methods. Here, Runge-Kutta Method is going to be used since it is a one step method obtained from the Taylor series expansion of $u$ up to and including the terms involving $(\Delta t)^{4}$ where $\Delta t$ is the step size with respect to time. The $4^{\text {th }}$ order RKM since its stability region is larger comparing to the other time integration methods and simple for the computations.

The resulting algebraic system of equation (29) can originally be considered as an initial value problem in the form (a set of ordinary differential equations in time)

$$
\{b\}=\frac{d\{u\}}{d t}=[A]\{u\}
$$

Thus the $4^{\text {th }}$ order RKM gives for the governing equation the 
following vector equation

$$
\left\{u_{n+1}\right\}=\left\{u_{n}\right\}+\frac{\Delta t}{6}\left[\left\{K_{1}\right\}+2\left\{K_{2}\right\}+2\left\{K_{3}\right\}+\left\{K_{4}\right\}\right]
$$

Where;

$$
\begin{aligned}
& K_{1}=f\left(t_{n}, u_{n}\right), \\
& K_{2}=f\left(t_{n}+\frac{\Delta t}{2}, u_{n}+\frac{\Delta t}{2} K_{1}\right), \\
& K_{3}=f\left(t_{n}+\frac{\Delta t}{2}, u_{n}+\frac{\Delta t}{2} K_{2}\right), \\
& K_{4}=f\left(t_{n}+\Delta t, u_{n}+\Delta t K_{3}\right),
\end{aligned}
$$

Applying $4^{\text {th }}$ order RKM equation (31) in equation (30). Thus, we can easily write by taking $[A]\{u\}$ as the vector function $\{f(t,\{u\})\}$ in the sample initial value problem $\dot{u}=f(t, u)$ So,

$$
\{f(t,\{u\})\}=[A]\{u\}
$$

The Matlab program has been used to solve this problem and get the velocity distribution.

\section{Numerical results and discussion}

We studied the unsteady pulsating flow of an incompressible couple stress fluid between permeable beds through Porous Medium in channel under the influence of periodic body acceleration in the presence of magnetic field numerical using new algorithm modified DQM. The algorithm is coded in Matlab 7.14.0.739 and the simulations are run on a Pentium 4 CPU 900 MHz with 1 GB memory capacity. We have shown the relation between the different parameters of motion such as Hartmann number $\mathrm{Ha}$, Couple stress parameter $\beta$, Reynolds number $R$, Knudsen number $k_{n}$, the permeability parameter of porous medium $k$ with the velocity distribution to investigate the effect of changing these parameters 
on the flow. Hence, we can be controlling the process of flow.

A numerical code has been written to calculate the velocity distribution according to equation (29).

Figs. 2, shows the variation of the velocity distribution with respect to Couple stress parameter $\beta$. It is seen that as $\beta$ increases (i.e., as Coefficient of couple stress viscosity $\eta$ decreases) the unsteady velocity increases. As $\beta \rightarrow \infty$ (i.e., as $\eta \rightarrow 0$ ), the velocity corresponds to non polar fluid.

Figs. 3, shows the variation of the velocity distribution with respect to the Hartmann number $\mathrm{Ha}$. It is seen that as $\mathrm{Ha}$ increases the unsteady velocity decreases.

Figs. 4, shows the variation of the velocity distribution with respect to the permeability parameter of porous medium $k$. It is seen that as $k$ increases the unsteady velocity increases.

Figs. 5, shows the variation of the velocity distribution with respect to the Reynolds number $R$ have less influence on the unsteady velocity. It is seen that as $R$ increases the unsteady velocity decreases. The decrease in velocity for each $R$ is clearly visible for the region $y=0$ to $y=0.8$. As $y=0.8$ to $y=1.0$, the velocity profile are almost coinciding for each $R$.

Figs. 6, shows that the variation of the velocity distribution with respect to the Knudsen number $k n$. It is clearly visible that the velocity increases with increasing $k n$ for the region $y=0$ to $y=[0.4: 0.5]$. As $y=0.5$ to $y=1.0$, the velocity decrease with increasing $k n$.

Figs. 7, shows the effects of Reynolds number $R$ with the velocity distribution for non polar viscous fluid $(\beta \rightarrow \infty$ i.e., $\eta \rightarrow 0$ ). As Reynolds number $R$ is increasing the velocity at any $y$ is decreasing. For $R=0$, the maximum velocity is attained exactly midway between the lower and upper permeable beds. As $R$ increases, the maximum velocity is attained 
nearer to the upper permeable bed.

Figs. 8, shows the effects of the permeability parameter of porous medium $k$ with the velocity distribution for non polar viscous fluid $(\beta \rightarrow \infty$ i.e., $\eta \rightarrow 0)$. As $k$ is increasing the velocity at any $y$ is increasing. The velocity profile corresponding to $R=2$. the maximum velocity nearer to the upper plate slightly (slowly).

Figs. 9, shows the effects of the Knudsen number $k n$ with the velocity distribution for non polar viscous fluid $(\beta \rightarrow \infty$ i.e., $\eta \rightarrow 0$ ). As the Knudsen number $k n$ is increasing for the region $y=0$ to $y=[0.4: 0.5]$. As As $y=0.5$ to $y=1.0$, the velocity decrease with increasing $k n$. The velocity profile corresponding to $R=2$. Here again the maximum velocity nearer to the upper plate slightly (slowly).

Figs. 10, 11, shows the effects of the Hartmann number $\mathrm{Ha}$ with the velocity distribution for non polar viscous fluid $(\beta \rightarrow \infty$ i.e., $\eta \rightarrow 0$ ). As $\mathrm{Ha}$ is increasing the velocity at any $\mathrm{y}$ is decreasing. The velocity profile corresponding to $R=2$. The maximum velocity region increase with increase the Hartmann number $\mathrm{Ha}$.

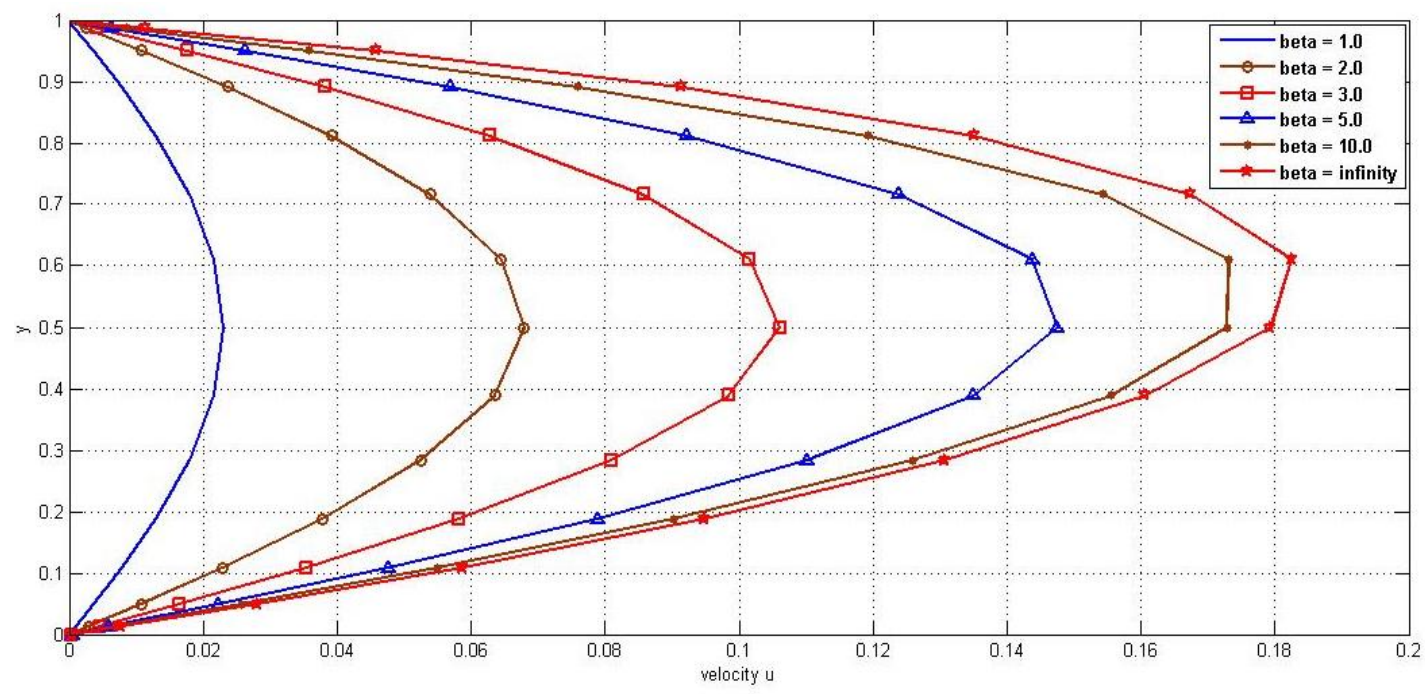

Fig. 2 The effect of Couple stress parameter on velocity distribution $[\mathrm{Ha}=1$, $\left.\mathrm{kn}=0.001, \mathrm{k}=0.5, \mathrm{R}=2, \mathrm{~A}_{\mathrm{O}}=2, \mathrm{~A}_{1}=1, \mathrm{a}_{\mathrm{O}}=1, \mathrm{~b}=2\right]$. 


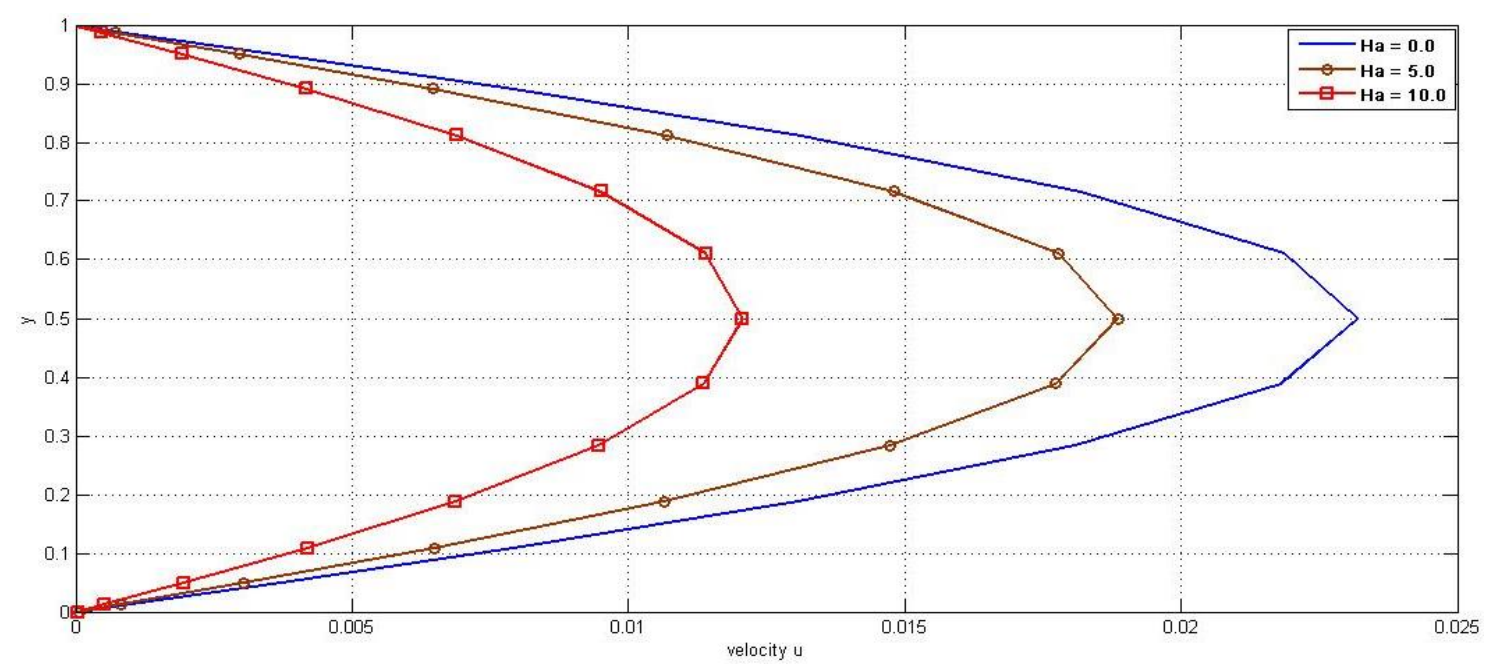

Fig. 3 The effect of Hartman number on velocity distribution $[k=0.5, R=2$, $\left.\mathrm{kn}=0.001, \beta=1, \mathrm{~A}_{\mathrm{O}}=2, \mathrm{~A}_{1}=1, \mathrm{a}_{\mathrm{O}}=1, \mathrm{~b}=2\right]$.

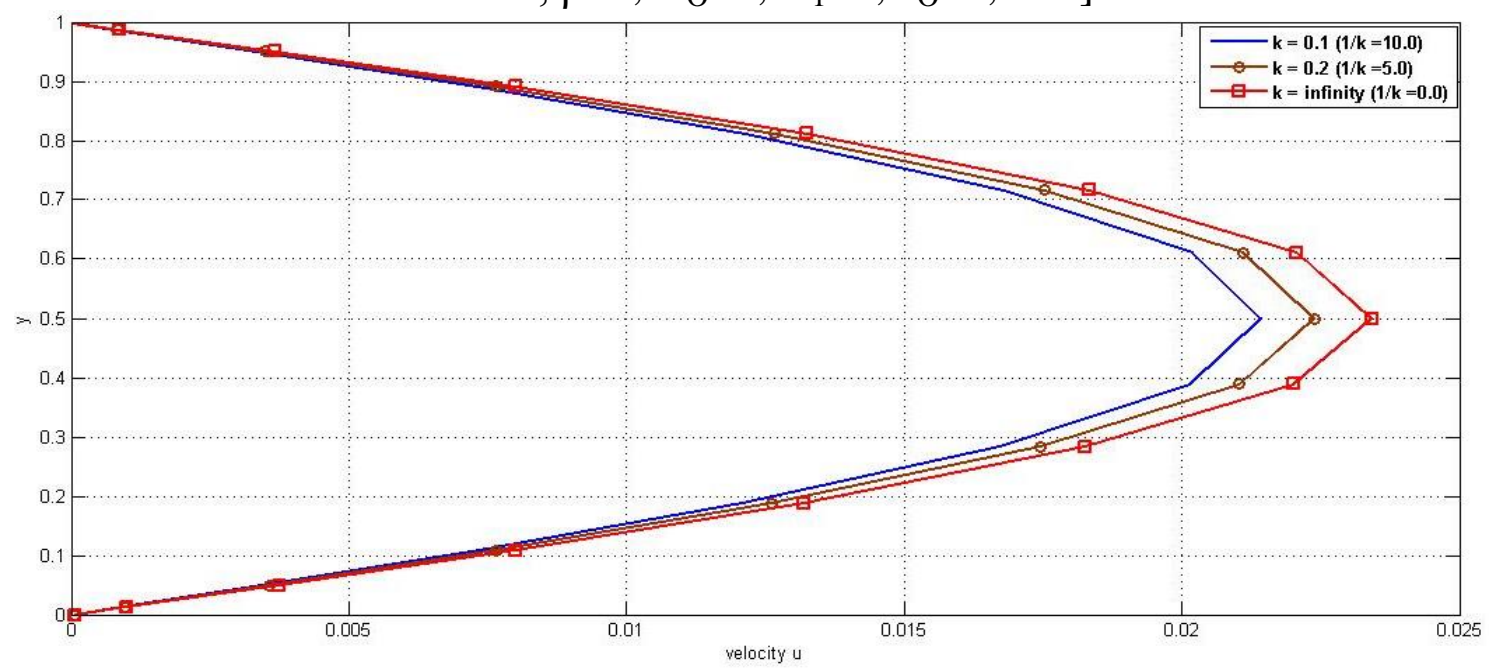

Fig. 4 The effect of Porosity parameter on velocity distribution $[\mathrm{Ha}=1, \mathrm{R}=2$,

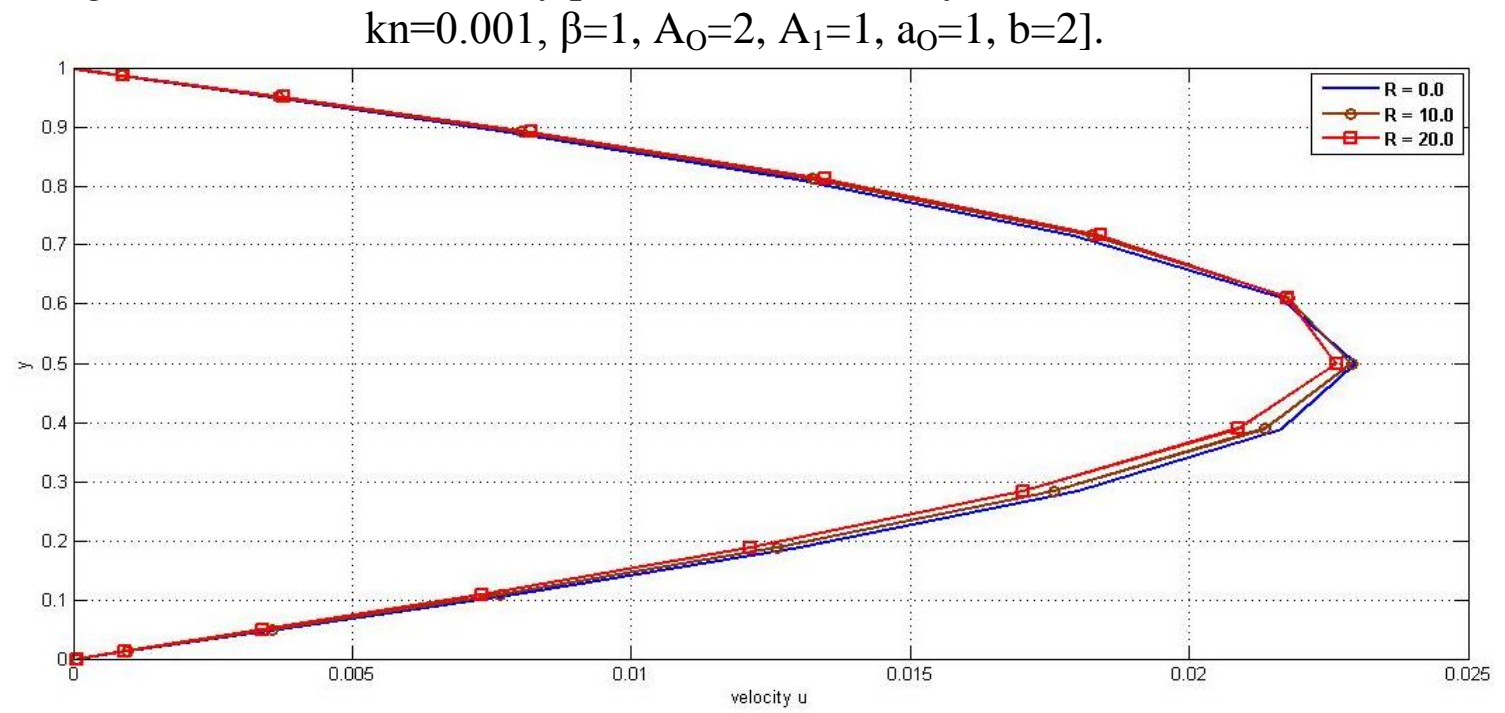

Fig. 5 The effect of Reynolds number on velocity distribution $[\mathrm{Ha}=1, \mathrm{k}=0.5$, $\mathrm{kn}=0.001, \beta=1, \mathrm{AO}=2, \mathrm{~A} 1=1, \mathrm{aO}=1, \mathrm{~b}=2]$. 


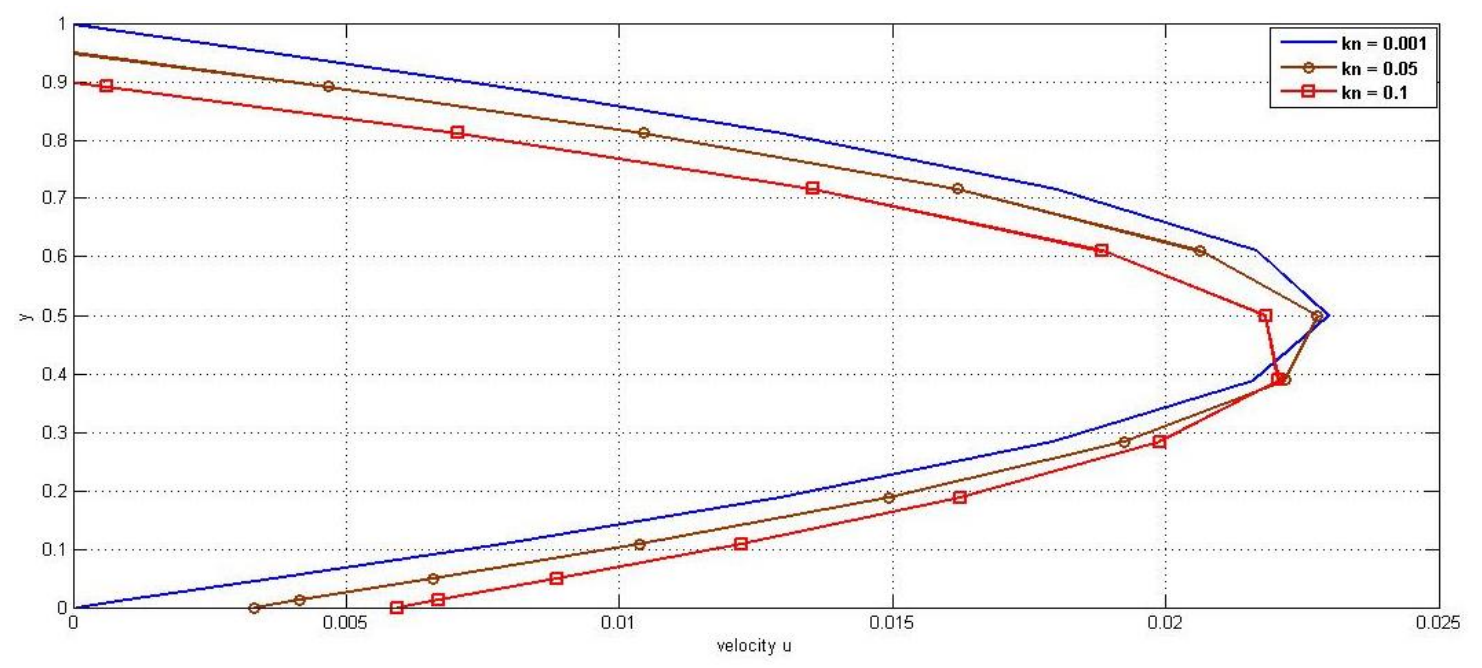

Fig. 6 The effect of Knudsen number on velocity distribution $[\mathrm{Ha}=1, \mathrm{k}=0.5$, $\left.\mathrm{R}=2, \beta=1, \mathrm{~A}_{\mathrm{O}}=2, \mathrm{~A}_{1}=1, \mathrm{a}_{\mathrm{O}}=1, \mathrm{~b}=2\right]$.

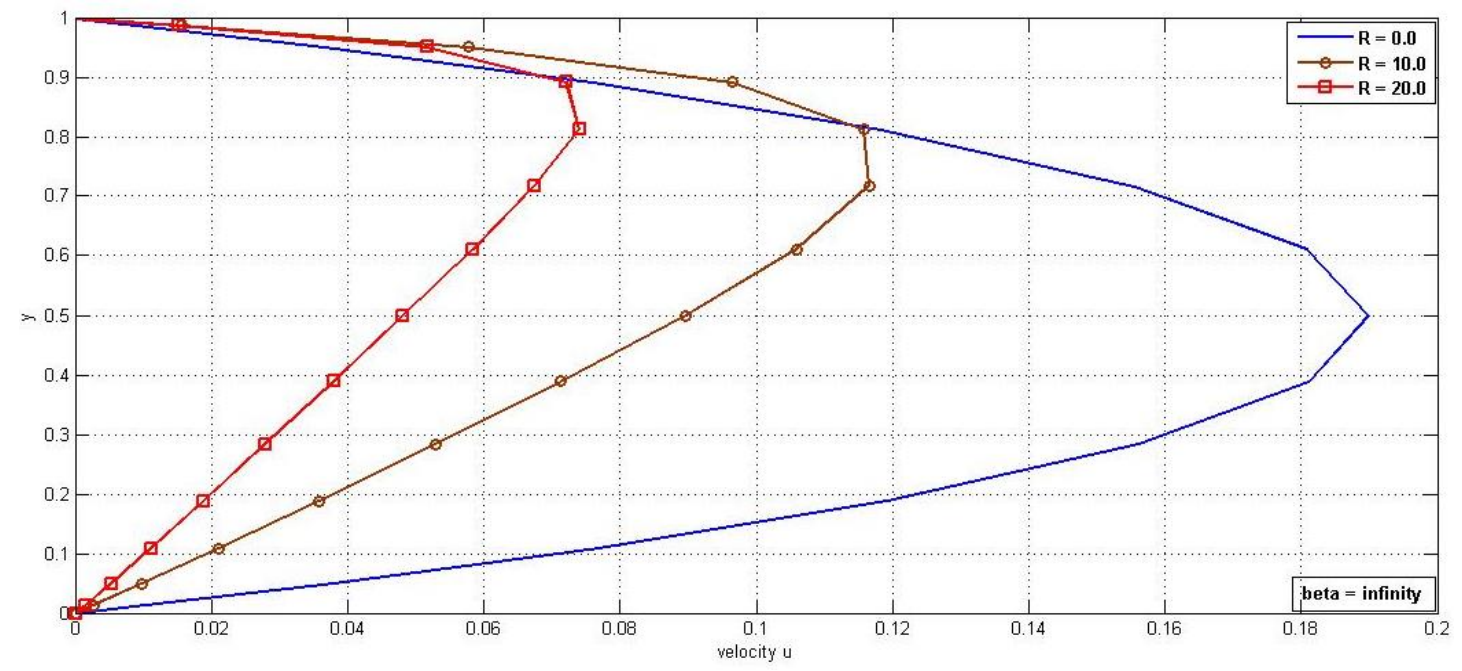

Fig. 7 The effect of Reynolds number on velocity distribution $[\mathrm{Ha}=1, \mathrm{k}=0.5$, $\left.\mathrm{kn}=0.001, \mathrm{~A}_{\mathrm{O}}=2, \mathrm{~A}_{1}=1, \mathrm{a}_{\mathrm{O}}=1, \mathrm{~b}=2, \beta \rightarrow \infty(\eta \rightarrow 0)\right]$.

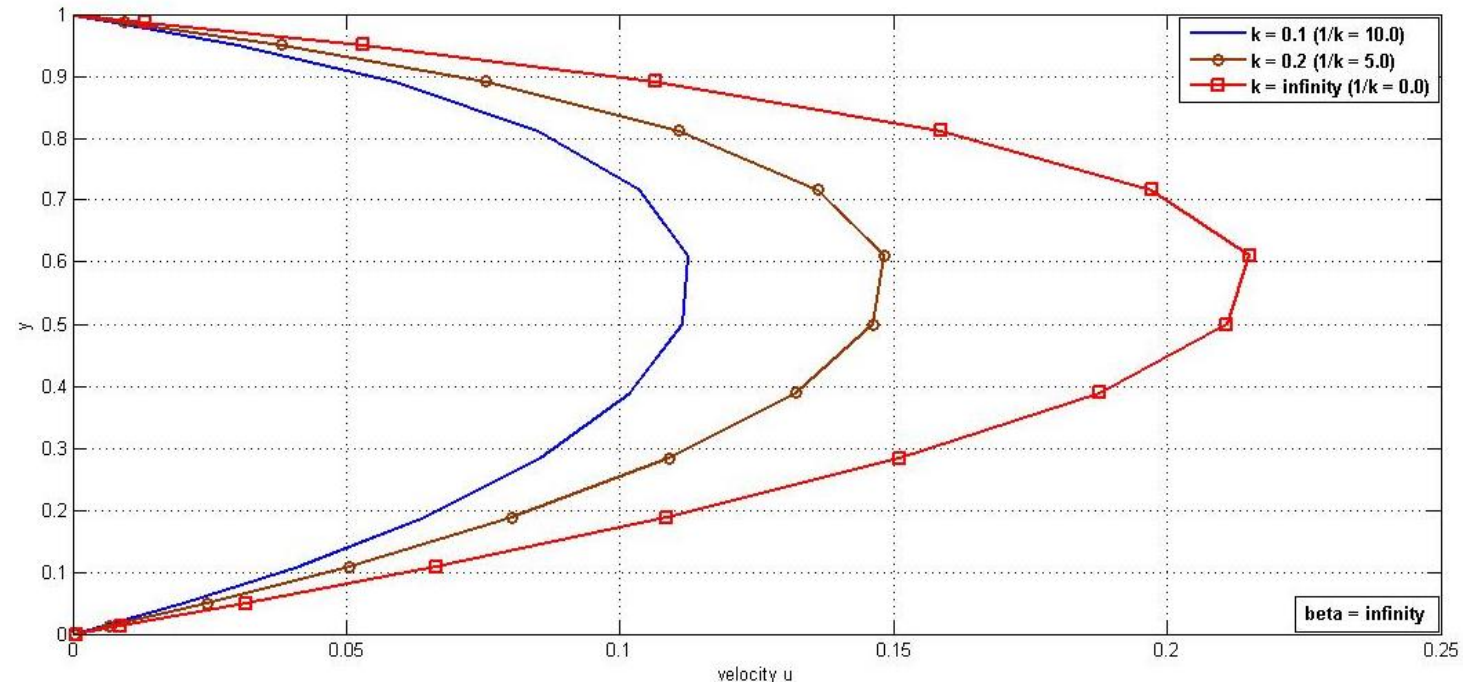

Fig. 8 The effect of Porosity parameter on velocity distribution $[\mathrm{Ha}=1, \mathrm{R}=2$, $\left.\mathrm{kn}=0.001, \mathrm{~A}_{\mathrm{O}}=2, \mathrm{~A}_{1}=1, \mathrm{a}_{\mathrm{O}}=1, \mathrm{~b}=2, \beta \rightarrow \infty(\eta \rightarrow 0)\right]$. 


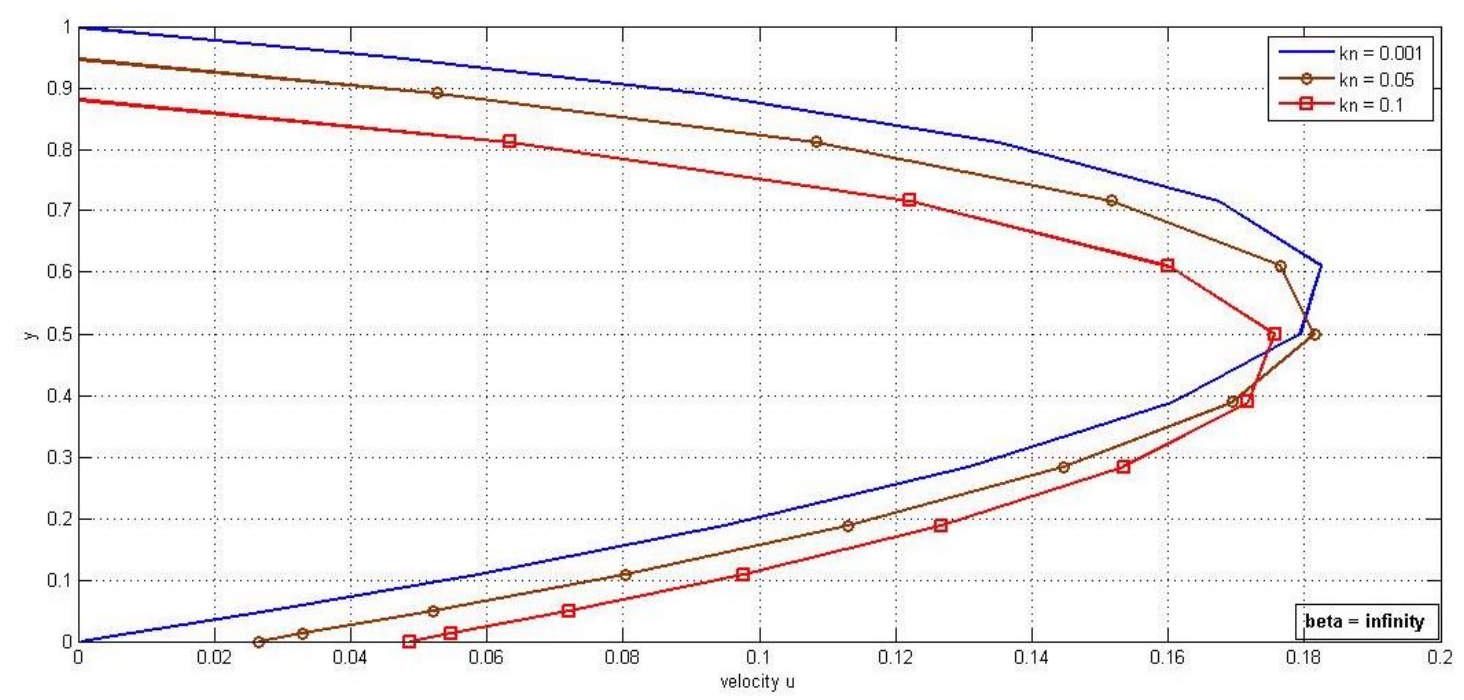

Fig. 9 The effect of Knudsen number on velocity distribution $[\mathrm{Ha}=1, \mathrm{R}=2$,

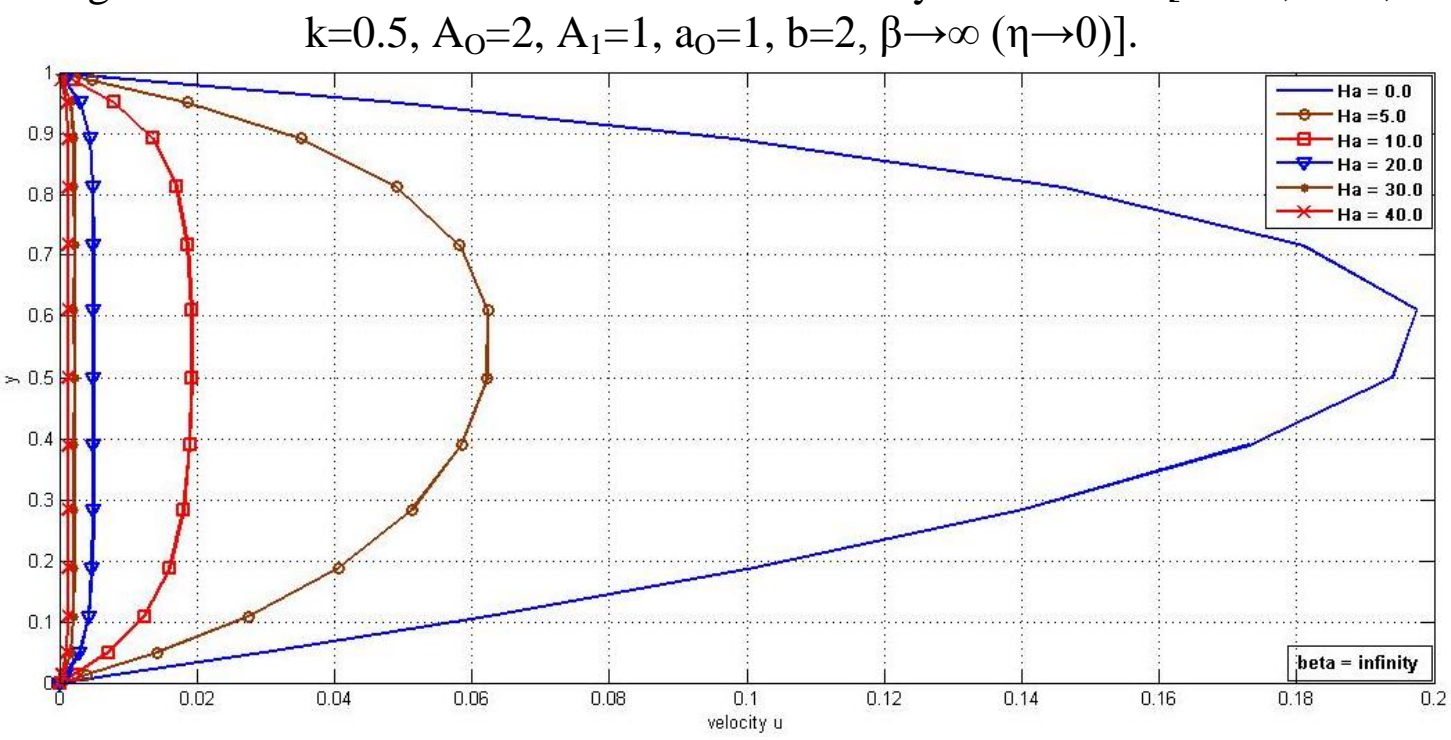

Fig. 10 The effect of Hartman number on velocity distribution $[k=0.5, R=2$,

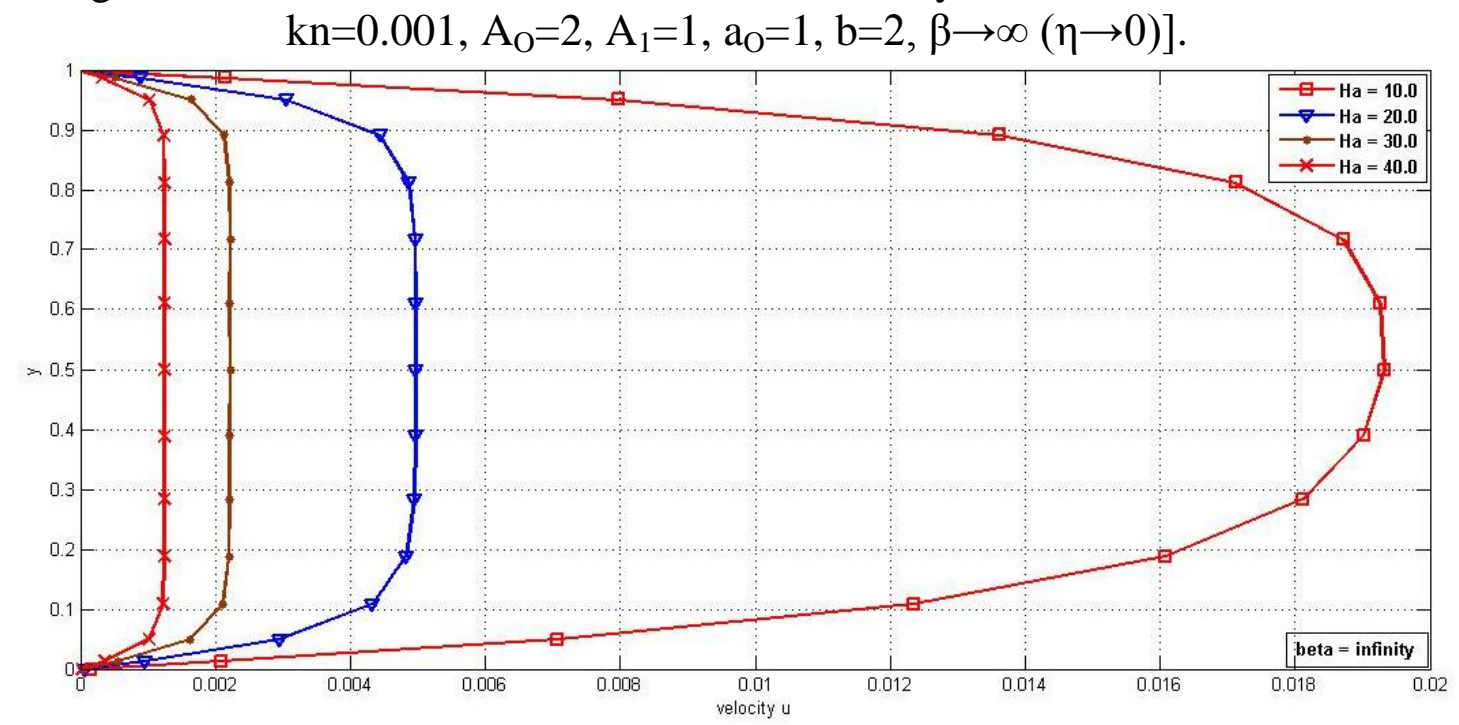

Fig. 11 The effect of Hartman number on velocity distribution $[k=0.5, R=2$, $\left.\mathrm{kn}=0.001, \mathrm{~A}_{\mathrm{O}}=2, \mathrm{~A}_{1}=1, \mathrm{a}_{\mathrm{O}}=1, \mathrm{~b}=2, \beta \rightarrow \infty(\eta \rightarrow 0)\right]$. 


\section{CONCLUSIONS}

We have studied the unsteady pulsating flow of an incompressible couple stress fluid between permeable beds through Porous Medium in channel under the influence of periodic body acceleration in the presence of magnetic field using a new algorithm modified GDQM. The slip condition on the wall artery has been considered.

The velocity has been obtained numerically. It is of interest to note that the unsteady velocity increases with increasing of the Couple stress parameter $\beta$ and the permeability parameter of porous medium $k$ whereas it decreases with increasing the Reynolds number $R$ and the Hartmann number $\mathrm{Ha}$. Also the velocity increases with increasing the Knudsen number $k n$ for the region $y=0$ to $y=[0.4: 0.5]$. As $y=0.5$ to $y=1.0$, the velocity decrease with increasing $k n$.

It is observed that the presence of couple stresses results in a decrease in the velocity. Also it is observed that when the couple stresses are present, the Reynolds number seems to have no influence on the unsteady velocity component. This is in contrast with the disturbance we see in the absence of couple stresses.

It is observed that when the couple stresses are present:

- As the Reynolds number $R$ increases, the maximum velocity is attained nearer to the upper permeable bed plate.

- As the Knudsen number $k n$ decreases, the maximum velocity is attained nearing to the upper permeable bed plate slightly (slowly).

- As the Hartmann number $H a$ increases, the maximum velocity region increase.

- As the permeability parameter of porous medium $k$ increases, the maximum velocity region increase. 


\section{APPENDIX}

$$
\begin{aligned}
& R_{1}=1-k_{n} A_{1,1}, \quad R_{2}=k_{n}^{2} A_{N, 1} \\
& R_{3}=1-k_{n} A_{N, N}-k_{n} A_{1,1}+k_{n}^{2} A_{1,1} A_{N, N}-k_{n}^{2} A_{N, 1} A_{1, N} \\
& R_{4}=A_{N, 1} k_{n}, \\
& R_{5}=\frac{R_{2}}{R_{3}}, \quad R_{6}=\frac{R_{1} R_{2}}{R_{3} R_{4}} \\
& R_{7}=\frac{k_{n}+k_{n} A_{1, N} R_{5}}{R_{1}}, \quad R_{8}=\frac{k_{n} A_{1, N} R_{6}}{R_{1}} \\
& R_{9}=B_{1,1} R_{7} A_{1,2}+B_{1,1} R_{8} A_{N, 2}+B_{1,2}+B_{1, N} R_{5} A_{1,2}+B_{1, N} R_{6} A_{N, 2} \\
& R_{10}=B_{1,1} R_{7}+B_{1, N} R_{5}, \quad R_{11}=B_{1,1} R_{8}+B_{1, N} R_{6} \\
& R_{12}=B_{1,1} R_{7} A_{1, N-1}+B_{1,1} R_{8} A_{N, N-1}+B_{1, N-1}+B_{1, N} R_{5} A_{1, N-1}+B_{1, N} R_{6} A_{N, N-1} \\
& R_{13}=B_{N, 1} R_{7} A_{1,2}+B_{N, 1} R_{8} A_{N, 2}+B_{N, 2}+B_{N, N} R_{5} A_{1,2}+B_{N, N} R_{6} A_{N, 2} \\
& R_{14}=B_{N, 1} R_{7}+B_{N, N} R_{5}, \quad R_{15}=B_{N, 1} R_{8}+B_{N, N} R_{6} \\
& R_{16}=B_{N, 1} R_{7} A_{1, N-1}+B_{N, 1} R_{8} A_{N, N-1}+B_{N, N-1}+B_{N, N} R_{5} A_{1, N-1}+B_{N, N} R_{6} A_{N, N-1} \\
& R_{17}=R_{13} R_{12}-R_{16} R_{9}, \quad R_{18}=R_{14} R_{9}-R_{13} R_{10} \\
& R_{19}=R_{15} R_{9}-R_{13} R_{11} \\
& R_{20}=\frac{R_{18}}{R_{17}}, \quad R_{21}=\frac{R_{19}}{R_{17}}, \quad R_{22}=-\frac{R_{13}}{R_{17}}, \quad R_{23}=-\frac{R_{9}}{R_{17}} \\
& R_{24}=-\frac{R_{10}+R_{12} R_{20}}{R_{9}}, \quad R_{25}=\frac{-1+R_{12} R_{22}}{R_{9}} \\
& R_{26}=-\frac{R_{11}+R_{12} R_{21}}{R_{9}}, \quad R_{27}=-\frac{R_{12} R_{23}}{R_{9}} \\
& R_{28}=R_{24}\left[R_{7} A_{12}+R_{8} A_{N, 2}\right], \quad R_{29}=R_{25}\left[R_{7} A_{12}+R_{8} A_{N, 2}\right] \\
& R_{30}=R_{26}\left[R_{7} A_{12}+R_{8} A_{N, 2}\right], \quad R_{31}=R_{27}\left[R_{7} A_{12}+R_{8} A_{N, 2}\right] \\
& R_{32}=R_{20}\left[R_{7} A_{1, N-1}+R_{8} A_{N, N-1}\right], \quad R_{33}=R_{21}\left[R_{7} A_{1, N-1}+R_{8} A_{N, N-1}\right] \\
& R_{34}=-R_{22}\left[R_{7} A_{1, N-1}+R_{8} A_{N, N-1}\right], \quad R_{35}=R_{23}\left[R_{7} A_{1, N-1}+R_{8} A_{N, N-1}\right]
\end{aligned}
$$




$$
\begin{array}{ll}
R_{36}=R_{28}+R_{7}+R_{32}, & R_{37}=R_{30}+R_{8}+R_{33} \\
R_{38}=R_{29}+R_{34}, & R_{39}=R_{31}+R_{35} \\
R_{40}=R_{24}\left[R_{5} A_{1,2}+R_{6} A_{N, 2}\right], & R_{41}=R_{25}\left[R_{5} A_{1,2}+R_{6} A_{N, 2}\right] \\
R_{42}=R_{26}\left[R_{5} A_{1,2}+R_{6} A_{N, 2}\right], & R_{43}=R_{27}\left[R_{5} A_{1,2}+R_{6} A_{N, 2}\right] \\
R_{44}=R_{20}\left[R_{5} A_{1, N-1}+R_{6} A_{N, N-1}\right], & R_{45}=R_{21}\left[R_{5} A_{1, N-1}+R_{6} A_{N, N-1}\right] \\
R_{46}=-R_{22}\left[R_{5} A_{1, N-1}+R_{6} A_{N, N-1}\right], & R_{47}=R_{23}\left[R_{5} A_{1, N-1}+R_{6} A_{N, N-1}\right] \\
R_{48}=R_{40}+R_{5}+R_{44}, & R_{49}=R_{42}+R_{6}+R_{45} \\
R_{50}=R_{41}+R_{46}, & R_{51}=R_{43}+R_{47}
\end{array}
$$

\section{REFERENCES}

[1] D. N. Ku, D. P. Giddens, C. K. Zairns, S. Glagov, "Pulsatile flow and atherosclerosis in human carotid bifurcation: positive correlation between plaque location and low and oscillating shear stress", Arteriosclerosis 5 293-302, 1985.

[2] R. M. Nerem, M. J. Levesque, "Hemodynamics and the arterial wall", Vasc. Disc. 295-317, 1987.

[3] F. Fedele, D. L. Hitt, R. D. Prabhu, "Revisiting the stability of pulsatile pipe flow", European Journal of Mechanics B/Fluids Vol. 24 237-254, 2005.

[4] N. A. S. Afifi and N. S. Gad, "Interaction of peristaltic flow with pulsatile magneto-fluid through a porous medium", Acta Mechanica vol. 149 229-237, 2001.

[5] G. Ramamurthy and B. Shanker, "Magnetohydrodynamic Effects on Blood Flow through Porous Channel," Medical and Biological Engineering and Computing, vol. 32, no. 6, pp. 655-659, 1994. 
[6] K. Das and G. C. Saha, "Arterial MHD Pulsatile Flow of Blood under Periodic Body Acceleration," Bulletin of Society of Mathematicians Banja Luka, vol. 16, pp. 21-42, 2009.

[7] C. S. Dulal and B. Ananda, "Pulsatile Motion of Blood through an Axi-Symmetric Artery in Presence of Magnetic Field," Journal of Science and Technology of Assam University, vol. 5, no. 2, pp. 12-20, 2010.

[8] P. Chaturani and V. Palanisamy, "Pulsatile flow of blood with periodic body acceleration," International Journal of Engineering Science, Vol. 29 no. 1, pp. 113-121, 1991.

[9] I. M. Eldesoky, "Slip Effects on the Unsteady MHD Pulsatile Blood Flow through Porous Medium in an Artery under the Effect of Body Acceleration," International Journal of Mathematics and Mathematical Sciences, Vol. 2012, Article ID 860239, 2010.

[10] I. M. Eldesoky, "Influence of Slip Condition on Peristaltic Transport of a compressible Maxwell Fluid through porous Medium in a tube," International Journal of Applied Mathematics and Mechanics, Vol. 8, pp. 99-117, 2012.

[11] I. M. Eldesoky, M. H. Kamel, Reda M. Hussien and Ramzy M. Abumandour, "Numerical Study of Unsteady MHD Pulsatile Flow through Porous Medium in an Artery Using Generalized Differential Quadrature Method (GDQM)". International Journal of Materials, Mechanics and Manufacturing, Vol. 1, No. 2 April 2013.

[12] I. M. Eldesoky, "Effect of Relaxation Time on MHD Pulsatile Flow of Blood through Porous Medium in an Artery under the Effect of Periodic Body Acceleration," Journal of Biological Systems, Vol. 21, Issue 2, pp. (1-17), 2013.

[13] Y. C. Wang, Pulsatile flow in a porous channel, Journal of Appllied Mechanics Vol. 38 553-555, 1972. 
[14] K. Vajravelu, K. Ramesh, S. Sreenadh, P.V. Arunachalam, Pulsatile flow between permeable beds., International Journal of Non-Linear Mechanics, Vol. 38, No. 7, pp. 999-1005, October 2003.

[15] G. S. Beavers, D. D. Joseph, Boundary conditions at a naturally permeable wall., Journal of Fluid Mechanics, Vol 30, pp. 197-207, . doi:10.1017/S00221120670013751967.

[16] V. K. Stokes, "Couple Stresses in Fluids", the Physics of Fluids, Vol. 9 pp. 1709-1715, 1966.

[17] V. K. Stokes, "Theories of Fluids with Microstructure", SpringerVerlag, Berlin 1984.

[18] N. B. Naduvinamani, P. S. Hiremath, G. Gurubasavaraj, "Squeeze film lubrication of a short porous journal bearing with couple stress fluids.", Tribology International, Vol. 34 no. 11, pp. 739-747, DOI: 10.1016/S0301-679X(01)00064-0, 2001.

[19] N. B. Naduvinamani, P. S. Hiremath, G. Gurubasavaraj, "Surface roughness effects in a short porous journal bearing with a couple stress fluid", Fluid Dynamics Research, 31(5-6), pp. 333-354, 2002.

[20] N. B. Naduvinamani, P. S. Hiremath, G. Gurubasavaraj, "Effects of surface roughness on the couple stress squeeze film between a sphere and a flat Plate.", Tribology International, Vol. 38 no. 5, pp. 451-458, 2005.

[21] N. B. Naduvinamani, Syeda Tasneem Fathima, P. S. Hiremath, "Hydrodynamic lubrication of rough slider bearings with couple stress fluids", Tribology International, Vol. 36 no. 12, pp. 949-959, 2003.

[22] N. B. Naduvinamani, Syeda Tasneem Fathima, P.S. Hiremath, "Effect of surface roughness on characteristics of couplestress squeeze film between anisotropic porous rectangular plates", Fluid Dynamics Research, Vol. 32 no. 5, pp. 217-231, 2003.

[23] J.-R. Lin, C.-R. Hung, Combined effects of non-Newtonian couple stresses and fluid inertia on the squeeze film characteristics between a 
long cylinder and an infinite plate, Fluid Dynamics Research, Vol. 39 no. 8, pp. 616-639, 2007.

[24] S. K. Lakshmana Rao and T. K. V. Iyengar, "Analytical and computational studies in couple stress fluid flows", U.G.C. Research project C-8-4/82 SR III, 1985.

[25] D. Srinivasacharya, "Stokes flow of an incompressible Couple stress fluid past an approximate sphere", Ph.D thesis, 1995.

[26] N. T. M. EL-Dabe, S. M. G. EL-Mohandis, "Effect of couple stresses on pulsatile hydromagnetic Poiseuille flow", Fluid Dynamics Research, Vol. 15(5), pp. 313-324, 1995.

[27] L. M. Srivastava, "Flow of couple stresss fluid through stenotic blood vessels", Journal of Biomechanics, Vol 18, 479-485, 1985.

[28] L. M. Srivastava, "Perstaltic transport of a couple stresss fluid", Rheologica Acta, Vol 25, 638-641, 1986.

[29] N. B. Naduvinamani, P. S. Hiremath and G. Gurubasavaraj, "Squeeze film lubrication of a short porous journal bearing with couple stress fluids", Tribology International,Vol 34, 739-747, 2001.

[30] N. B. Naduvinamani, P. S. Hiremath and G. Gurubasavaraj, "Surface roughness effects in a short porous journal bearing with couple stress fluid", Fluid Dynamics Research,Vol 31, 333-354, 2002.

[31] N. B. Naduvinamani, Syeda Taseem Fathima and P. S. Hiremath, "Effects of surface roughness on characteristics of couple stress squeeze film between anisotropic porous rectangular plates", Fluid Dynamics Research, Vol 32, 217-231, 2003.

[32] M. Devakar and T. K. V.Iyengar, "Stoke's problems for an incompressible couple stress fluid", Nonlinear Analysis: Modeling and Control, Vol 1, 181-190, 2008. 
[33] M. Devakar and T. K. V. Iyengar, "Runup flow a couple stress fluid between parallel plates", Nonlinear Analysis: Modeling and Control, Vol 15, 29-37, 2010.

[34] T. S. L. Radhika and T. K. V. Iyengar, "Stokes flow of an incompressible couple stress fluid past a porous spherical shell", Proceedings of International Multi conference of Engineers and Computer Scientists, Vol 3, 1634-1639, 2010.

[35] N. T. M. EL-Dabe, S. M. G. EL-Mohandis, Effect of couple stresses on pulsatile hydromagnetic Poiseuille flow, Fluid Dynamics Research, Vol. 15(5), pp. 313-324, 1995.

[36] R. E. Bellman and J. Casti, "Differential quadrature and long-term integration," Journal of Mathematical Analysis and Applications, Vol. 34, pp. 235-238, 1971.

[37] R. E. Bellman, B. G. Kashef and J. Casti, "Differential quadrature: A technique for the rapid solution of non-linear partial differential equations", Journal of computational Physics Vol. 10, pp. 40-52, 1972.

[38] S. K. Jang, C. W. Bert, and A. G. Striz, “Application of differential quadrature to static analysis of Structural components," International Journal of Numerical Methods in Engineering, vol. 28, pp. 561-577, 1989.

[39] C. Shu and B. E. Richards, "High resolution of natural convection in a square cavity by generalized differential quadrature", Proceedings of $3^{\text {rd }}$ International Conference on Advanced in numerical Methods in Engineering: Theory and Applications, Swansea, U.K 2: 978-985, 1990.

[40] C. Shu, "Generalized differential-integral quadrature and application to the simulation of incompressible viscous flows including parallel computation", PhD thesis, Department of Aerospace Engineering, University of Glasgow, 1991. 
[41] C. Shu and B. E. Richards, "Application of generalized differential quadrature to solve two-dimensional incompressible Navier-Stokes equations," International Journal of Numerical Methods in Fluid Dynamics, Vol. 15, pp. 791-798, 1992.

[42] C. Shu, "Differential quadrature and its application in engineering", Springer-Verlag, London, 2000.

[43] M. Tanaka and W. Chen, "Coupling dual reciprocity boundary element method and differential quadrature method for time dependent diffusion problems," Applied Mathematical Modelling, vol. 25, no. 3, pp. 257-268, 2001.

[44] P. Jun-ping, and Z. Jian-jun, "Structural Dynamic Responses Analysis Applying Differential Quadrature Method," Journal of Zhejiang University Science, vol. 7, no. 11, pp. 1831-1838, 2006.

[45] Zhi Zong and Yingyan Zhang, "Advanced Differential Quadrature Methods", Chapman \& Hall/CRC applied mathematics and nonlinear science series, 2009. 